\title{
Cataratas: Una rara complicación de quemaduras eléctricas*
}

\author{
Drs. MARÍA FRANCISCA NAVARRETE C. ${ }^{1}$, CARLOS PILASI M. ${ }^{1}$, \\ JUAN PABLO QUINTEROS P. ${ }^{1}$, WILFREDO CALDERÓN O. ${ }^{1}$ \\ 1 Servicio de Cirugía Plástica y Quemados del Hospital del Trabajador. \\ Santiago, Chile.
}

Abstract

\section{Cataracts: a rare complication of electric burns}

Introduction: Electric burns are complex lesions with multiple complications. Cataracts are an infrequent complication. There are several case reports, but very few information of its phisiopatology and clinical characteristics in the surgical literature. Objectives: To report the incidence and characteristics of patients admitted to Hospital del Trabajador de Santiago (HTS) with electric burns and secondary cataracts. And review the literature. Patients and Methods: All patients admitted to the HTS with electric burns between 01/01/1999 and 01/01/2009 were included. Their electronic files were reviewed, and their data were tabulated in an Excel sheet. The literature about electric burns and cataracts was reviewed. Results: There were 977 patients admitted with electric burns in the last 10 years, $3(0.3 \%)$ of them developed secondary cataracts. All were male, 33, 36 and 64 years old. Two of them had facial burns, only one had the description of the voltage $(13.500 \mathrm{v})$. The time between the burn and the diagnostic of cataract was 6 month, 2 and 4 years. In two cases the cataracts were bilateral and in one case it was unilateral and right sided. All patients received surgical treatment with satisfactory outcomes. Conclusions: Cataracts secondary to electric burns are infrequent. We need more numerous series, with accurate registers to establish some prognostic factors. It is important to have in mind this complication and actively look for it in patients that present with electric burns.

Key words: Electric burns, cataracts.

\section{Resumen}

Introducción: Las quemaduras eléctricas producen lesiones complejas con múltiples complicaciones. Las cataratas son una complicación infrecuente. Existen varios reportes de casos, pero escasa difusión en el ámbito quirúrgico de sus características y fisiopatología. Objetivo: Reportar la incidencia y características de la población atendida en el Hospital del Trabajador de Santiago (HTS) con diagnóstico de catarata secundaria a quemadura eléctrica, y presentar una revisión de la literatura sobre el tema. Pacientes y Métodos: Se incluyeron todos los pacientes admitidos al HTS con diagnóstico de quemadura eléctrica, entre el 01/01/1999 y el 01/01/2009. Se revisaron sus archivos electrónicos y se tabularon en una base de datos Excel. Se realizó una revisión sobre el tema. Resultados: En los últimos 10 años ingresaron 977 pacientes con diagnóstico de quemadura eléctrica, de ellos, $3(0,3 \%)$ tenían cataratas secundarias. Todos de sexo masculino, de 33, 36 y

\footnotetext{
*Recibido el 01 de Noviembre de 2009 y aceptado para publicación el 12 de Enero de 2010.

Correspondencia: Dra. María Francisca Navarrete C. Marcoleta 367, Santiago, Chile.

E-mail: fcanavarrete@gmail.com
} 
64 años. En dos de ellos se reportan quemaduras faciales al ingreso y sólo en uno se describe el voltaje de la quemadura $(13.500 \mathrm{v})$. El tiempo transcurrido entre la quemadura eléctrica y el diagnóstico de catarata fue de 6 meses, 2 y 4 años. En dos casos la catarata desarrollada fue bilateral y en un caso fue unilateral derecha. Todos los pacientes recibieron tratamiento quirúrgico con resultados satisfactorios. Conclusiones: Las cataratas secundarias a quemadura eléctrica son poco frecuentes. Hacen falta series más numerosas, con un buen registro, para poder establecer factores pronósticos. Es importante tener presente esta complicación y buscarla dirigidamente en los pacientes con quemadura eléctrica.

Palabras clave: Quemadura eléctrica, catarata.

\section{Introducción}

Las quemaduras eléctricas son lesiones complejas que pueden causar múltiples complicaciones, tanto en el punto de contacto con la fuente de electricidad como a distancia. Las cataratas son una complicación poco frecuente, pero conocida desde hace mucho tiempo, siendo descrita por primera vez por St. Ives, tras una lesión por rayo en $1722^{1,2}$.

La catarata es una opacidad del cristalino, de densidad variable y que puede deberse a una diversidad de causas. Las más frecuentes son las relacionadas al envejecimiento, con incidencias de hasta 50\% entre los 65 y 74 años (Figura 1). Las cataratas traumáticas son mucho menos frecuentes y pueden deberse a distintos mecanismos ${ }^{3}$.

La catarata traumática más frecuente es la secundaria a lesión por cuerpo extraño del cristalino o trauma contuso del globo ocular (Figura 2). Con menor frecuencia, otras causas incluyen, exposición excesiva al calor (catarata del soplador de vidrio), rayos $\mathrm{X}$, radioterapia, materiales radioactivos $\mathrm{y}$ electricidad ${ }^{3}$.

Existen varios reportes de casos que describen la incidencia de esta patología y caracterizan a la población afectada. La mayoría de ellos están publicados en revistas de Oftalmología y existe escasa

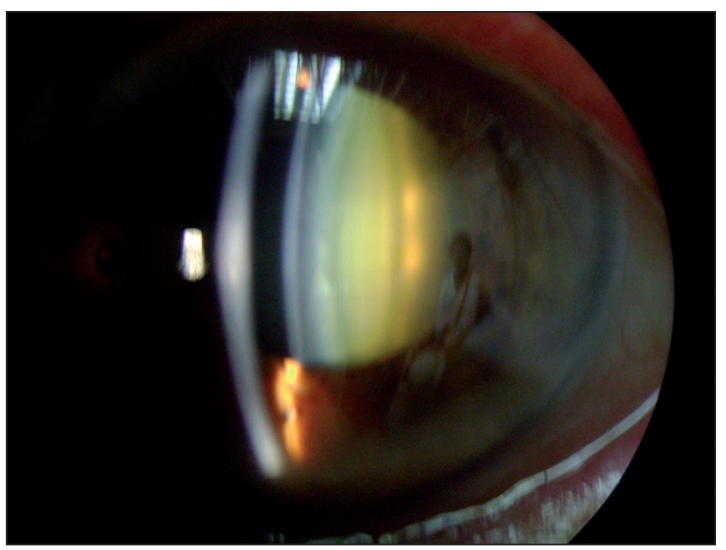

Figura 1. Catarata senil intermedia. difusión en el ámbito quirúrgico de las características y la fisiopatología de esta complicación.

\section{Material y Método}

Se incluyeron en el estudio todos los pacientes admitidos al Hospital del Trabajador de Santiago (HTS) con el diagnóstico de quemadura eléctrica, entre el $1^{\circ}$ de Enero de 1999 y el $1^{\circ}$ de Enero de 2009. Se revisaron los archivos electrónicos buscando pacientes con diagnóstico de quemadura eléctrica y catarata, y se tabularon los datos relevantes en una base de datos Excel. Paralelamente, se realizó una revisión de la literatura sobre el tema en PubMed y Medline, y se analizaron los datos obtenidos en nuestro centro en relación a las experiencias reportadas en la literatura.

\section{Resultados}

Entre el $1^{\circ}$ de Enero de 1999 y el $1^{\circ}$ de Enero de 2009, ingresaron al HTS 977 pacientes con diagnóstico de quemadura eléctrica, de los cuales 8 tenían también diagnóstico de catarata. De ellos, 4 correspondían a cataratas traumáticas (cáusticos, golpe directo), 1 a catarata senil y sólo 3 fueron

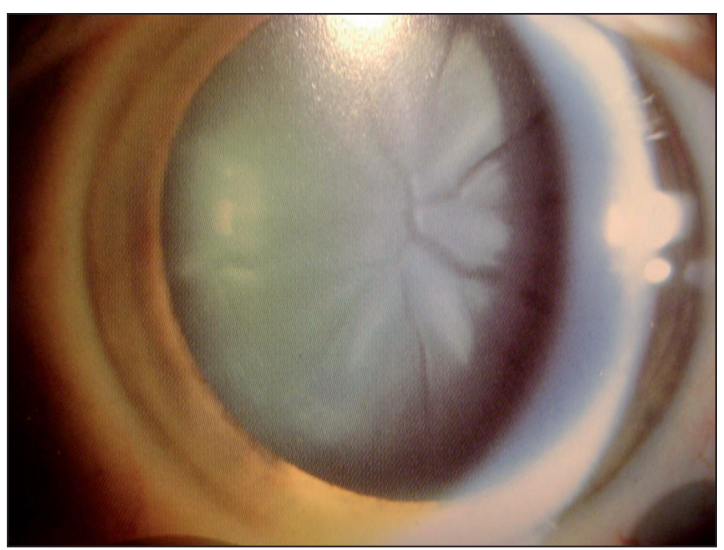

Figura 2. Catarata en flor o roseta, forma asociada a trauma ocular. 
diagnosticados como catarata secundaria a quemadura eléctrica, lo que corresponde a una incidencia de $0,3 \%$.

Los tres casos correspondían a pacientes de sexo masculino, de 33, 36 y 64 años de edad, en ningún caso se describen los puntos de entrada y salida de la corriente eléctrica, sin embargo, en dos de los casos existe registro de quemaduras faciales al ingreso. Sólo en uno de ellos se describe el voltaje y fue de 13.500 volts. La superficie corporal quemada al ingreso está descrita en dos de los casos y corresponden a 20 y $29 \%$.

El tiempo transcurrido entre la quemadura eléctrica y el diagnóstico de catarata fue de 6 meses, 2 años y 4 años, en cada caso.

En dos casos la catarata desarrollada fue bilateral y en un caso fue unilateral derecha. No disponemos del dato de la lateralidad del punto de entrada de la corriente, pero sabemos que este paciente presentaba quemaduras faciales al ingreso. Tras 4 años de seguimiento, el paciente no ha desarrollado catarata en el ojo contralateral.

Todas las cataratas recibieron tratamiento quirúrgico con resultados satisfactorios.

\section{Discusión}

La incidencia de las quemaduras eléctricas en Chile es aproximadamente de 4.5/100.000 habitantes por año y ha aumentado en los últimos años especialmente en el grupo en edad laboral activa ${ }^{4,5}$.

La diferencia entre quemaduras eléctricas de alto y bajo voltaje se hace arbitrariamente a los 1.000 volts. Las lesiones por quemadura con alto voltaje suelen causar destrucción en los puntos de contacto, con necrosis masiva de tejidos y daño en toda la vía de paso de la corriente, manifestándose con arritmias, alteraciones neurológicas o falla renal aguda, y secuelas como amputaciones, déficits neurológicos o cataratas ${ }^{6,7}$.

Se ha reportado aparición de cataratas después de diversos tipos de exposición a electricidad: cardioversión eléctrica, electrocución en silla eléctrica, terapia electroconvulsiva y quemadura eléctrica ${ }^{2,8}$.

La aparición de cataratas como consecuencia de electrocución severa, se reporta con una incidencia de hasta $5 \%$ en pacientes con puntos de entrada de la corriente sobre el cuello9. En nuestro centro, la incidencia de cataratas secundarias a quemadura eléctrica es bastante menor $(0,3 \%)$, pero por problemas de registro no conocemos los puntos de entrada y salida de la corriente en la gran mayoría de los pacientes con quemadura eléctrica.

Según Long, las cataratas aparecen más temprano y progresan más rápidamente en el ojo más proximal al lado de entrada de la corriente eléctrica. Y mientras más cerca es el punto de contacto de la corriente con el ojo, mayor el potencial de formar una catarata ${ }^{8,10}$.

Sin embargo, en el estudio de Ferreiro et al, reportan no haber encontrado ninguna relación entre el punto de entrada o la vía de tránsito de la electricidad y la incidencia de insuficiencia renal aguda, arritmias o cataratas 6 .

La latencia reportada en la aparición de la catarata varía desde inmediatamente después de la quemadura hasta años más tarde ${ }^{8}$. En nuestro centro la latencia encontrada fluctúa entre 6 meses y 4 años.

En cuanto a la morfología de las cataratas, en general, se caracterizan por edema del cristalino, alteración de proteínas, aumento de la proliferación y rotura de la continuidad de sus fibras. El edema del cristalino varía de acuerdo a la etapa de desarrollo de la catarata. La catarata inmadura (incipiente) es sólo ligeramente opaca, el cristalino con catarata madura es opaco por completo y moderadamente edematoso. Cuando el contenido de agua es máximo y se estira la cápsula del cristalino, la catarata se conoce como intumescente (hinchada). En la catarata hipermadura (muy avanzada) el agua se escapa del cristalino y deja un cristalino muy opaco, más o menos deshidratado, y una cápsula arrugada ${ }^{3}$.

Existen múltiples teorías respecto a la patogénesis de la catarata eléctrica, siendo la más aceptada, un cambio en la permeabilidad capsular que en sus primeras etapas se manifiesta por la aparición de múltiples pequeñas vacuolas justo debajo de la cápsula anterior. En pocos días a semanas, las vacuolas son reemplazadas por finas e irregulares opacidades subcapsulares anteriores ${ }^{8-10}$. Algunos autores afirman que una vez que las vacuolas se reabsorben hay proliferación de células sobre la porción anterior de los lentes. Como resultado del trauma eléctrico se alteran las capacidades metabólicas de las células no mitóticas de la zona central, adquiriendo capacidad de división y produciendo una sustancia similar a una cápsula. La duplicación local de la cápsula se correlaciona con el aspecto clínico de opacidades fibrilares anteriores ${ }^{11,12}$.

La fisiopatología de la catarata por cuerpo extraño es bastante más simple: el cristalino se vuelve blanco poco después de la penetración del cuerpo extraño, ya que la interrupción de su cápsula permite que el humor acuoso y a veces el vítreo penetren en la estructura del cristalino ${ }^{3}$.

Otros estudios similares al nuestro, muestran resultados muy variables: Saffle et al, revisaron 113 pacientes con lesiones eléctricas mayores e identificaron 7 (6,19\%) pacientes con cataratas. De ellos, 6 tenían cataratas bilaterales y sólo un paciente tenía una catarata unilateral ${ }^{1}$. 
Min HK et al, revisaron la casuística del Hanil General Hospital de Korea entre 1981 y 1993 y encontraron 663 pacientes con quemaduras eléctricas, de ellos $7(1,7 \%)$ desarrollaron cataratas. Todos tuvieron quemaduras de alto voltaje. El diagnóstico se hizo entre 2 y 18 meses después del accidente ${ }^{13}$.

Haberal $\mathrm{M}$ et al, realizaron un estudio epidemiológico en el Hospital de la Universidad de Hacettepe en Ankara, Turkia, entre los años 1979 y 1992, en que describen: 1.065 pacientes admitidos a la unidad de quemados, de ellos 219 (20,1\%) presentaban quemaduras eléctricas, de los cuales 4 presentaron complicaciones oculares: 2 keratoconjuntivitis y 2 desarrollaron cataratas $(0,9 \%)^{14}$.

\section{Conclusiones}

Las cataratas secundarias a quemadura eléctrica costituyen una complicación de baja frecuencia, que se ha intentado relacionar con múltiples factores predictivos. Se necesitan series más grandes, con un buen registro de los casos para poder hacer asociaciones que eventualmente sirvan como factores predictivos. Por lo tanto, es importante recordar que esta complicación existe y hay que buscarla dirigidamente en los pacientes que ingresan con diagnóstico de quemadura eléctrica.

\section{Agradecimientos}

Al Dr. Eugenio Álvarez Inostroza Residente de Oftalmología de la Pontificia Universidad Católica, quien revisó el trabajo y nos cedió las imágenes.

\section{Referencias}

1. Saffle J, Crandall A, Warden G. Cataracts: A Longterm Complication of Electrical Injury. The Journal of Trauma 1985; 25: 17-21.
2. Boozalis G, Purdue G, Hunt J, McCulley J. Ocular Changes from Electrical Burn Injuries. J Burn Care Rehabil 1991; 12: 458-462.

3. Shock JP, Harper RA. Cristalino. En: Vaughan DG, Asbury T, Riordan-Eva P. Oftalmología General, $11^{\circ}$ Edición, 1997, Editorial Manual Moderno, S.A. Capítulo 8, pg 191-201.

4. Sciaraffia C, Andrades P, Wisnia P. Quemaduras. En Andrades P, Sepúlveda S. Cirugía Plástica Esencial $1^{\circ}$ Edición, 2005, Editorial Universidad de Chile, Capítulo 7, pg 87-110.

5. Danilla S, Pastén JA, Fasce G, Díaz V, Iruretagoyena M. Mortality trends from Burn Injuries in Chile: 19541999. Burns 2004; 30: 348-356.

6. Ferreiro I, Meléndez J, Regalado J, Béjar FJ, Gabilondo FJ. Factors influencing the sequelae of high tensión electrical injuries. Burns 1998; 24: 649-653.

7. Luce EA. Electrical burns. Clin Plast Surg 2000; 27: 133-143.

8. Grewal DS, Jain R, Brar GS, Grewal SP. Unilateral electric cataract: Scheimpflug imaging review of the literature. J Cataract Refract Surg 2007; 33: 11161119.

9. Seth RK, Abedi G, Daccache AJ, Tsai JC. Cataract secondary to electrical shock from a Taser gun. J Cataract Refract Surg 2007; 33: 1664-1665.

10. Long JC. A Clinical and experimental study of electric cataract. Trans Am Ophthalmol Soc 1962; 60: 471-516.

11. Oleszewski SC, Nyman JS. Electric Cataract: A Rare Clinical Entity. American Journal of Optometry \& Physiological Optics 1984; 61: 279-283.

12. Hanna C, Fraunfelder FT. Electric cataracts: II. U1trastructural lens changes. Arch Ophthalmol 1972; 87: 184-191.

13. Min HK, Kim KH, Choi YI. Clinical Characteristics of Electric Cataract. J Korean Ophthalmol Soc 1995; 36: 1307-1311.

14. Haberal M, Uqar K, Bayraktar O, Oner R. Severe complications in electrical burns (Including visceral Complications). Annals of Burns and Fire Disasters 1996; 9: 233-239. 